\title{
XVIII.
}

\section{Otitis media acuta, ein Frühsymptom der Polyarthritis rheumatica acuta.}

\author{
Von \\ Dr. Oskar Wolf \\ in Frankfurt am Main.
}

Gelegentlich eines Vortrages über die Beziehungen der Ohrenkrankbeiten $\mathfrak{z u}$ den Allgemeinkrankheiten des Körpers 1) konnte ich darauf hinweisen, dass die sogenannte Sklerose der Paukenhöhlenauskleidung in zahlreichen Fällen mit der Polyarthritis rheumatica in ätiologischem Zusammenhang stehe. Ich musste mich bei dieser Annahme damals im Wesentlichen auf die Anamnese stititzen, denn Beobachtungen über die erste Entwicklung der Ohrenerkrankung während der acuten Periode der Polyarthritis lagen nicht vor. Erst in den letzten Jahren hatte ich Gelegenheit, diese erste Entstehung zu verfolgen. Wenn ich der vorliegenden Mittheilung den Titel gebe: „Otitis media acnta, ein Frubsymptom der Polyarthritis rheumatica acuta", so soll damit nur gesagt sein, dass die Ohrerkrankung zuweilen die erste Localisirung der rheumatischen Infection bildet, und dass dann erst später Gelenke, Pericardium, resp. Pleura ergriffen werden, während wieder in einer wahrscheinlich grösseren Anzahl von Fällen die Ohrerkrankung nach oder während der Gelenkaffectionen auftritt und dann oft ubersehen wird, weil sie nur selten die purulente Form annimmt und uberhaupt der schweren rheumatischen Allgemeinerkrankung gegenuber in den Hintergrund tritt. Wie man aus den unten folgenden beiden Krankengeschichten ersehen wird, so ist das Secret der Paukenhöhle bei dieser Form mehr schleimig-flockig, ähnlich demjenigen der erkrankten Gelenke; selten eitrig und zumeist wenig copiös, so dass es wahrscheinlich öfters uberhaupt nicht bemerkt worden ist. Die Auskleidung der Paukenhöhle ist histologisch nicht wesentlich verschieden von derjenigen der Gelenke, und die Gehörknöchelchen baben auch Gelenkverbindungen; es ist deshalb gar nicht zu verwundern,

1) Tageblatt der 59. Versammlung deutscher Naturforscher und Aerzte zu Wiesbaden 1887. 
dass die rhenmatische Infection, deren Träger allerdings bis jetzt noch nicht entdeckt sind, anch die Paukenhöble befällt. Aber nicht nur flir die Aetiologie der Sklerose der Paukenböhle und der Rigidität der Gehörknöchelchenkette ist es wichtig; diese rheumatisehe Form zu kennen, auch zur Vermeidung prognostischer Irrungen sind die beiden Fälle, welche ich hier einftige, gewissermaassen als Typen lehrreich.

F. Sp., 50 Jahre alter Kaufmann, erkrankte am 8. Juni 1888 mit Schmerz im linken Obre und Schwerhörigkeit. Ich fand die Symptome einer subacuten Otitis media und stand, da die Schmerzen auf Cataplasmen bald nachliessen, zunächst von der Paracentese ab. Am dritten Tage entleerte sich ohne erheblichen Schmerz schleimig flockiges Secret, und die Hörweite hob sich. Am vierten Tage erkrankte das rechte Ohr unter ähnlichen Symptomen; nach der Paracentese des Trommelfelles entleerte sich Anfangs blutig seröses, später schleimig flockiges Secret, und es trat Erleichterung ein. Die Körpertemperatur stieg jedoch am Abend des fünften Tages auf $39,6^{\circ} \mathrm{O}$, am Abend des sechsten auf $39,2^{\circ} \mathrm{C}$. Dazu traten Koptschmerzen, Gliederschmerzen, Appetitlosigkeit und Prostration des Kräftezustandes. Der Zustand des Patienten war besorgnisserregend; am siebenten Tage erschienen dann die Symptome des acuten Rbeumatismus, indem beide Metatarsalgelenke zu schwellen anfingen und schmerzhaft wurden. Die Krankheit wurde im weiteren Verlaufe eine sehr schwere, indem viele Gelenke nach einander befallen wurden, und Endocarditis hinzutrat. Die Paukenhöhlen hellten in der vierten Woche aus, es blieb nur massige Verdickung der Auskleidung und Verminderung der Hörweite etwa bis zur Hälfte der normalen zurück. Der Kranke erlag $11 / 2$ Jahre später den Folgen seiner Endocarditis.

Ad o If M. Bis dahin gesunder, 18 Jahre alter Gärtoer, erkrankte am 1. April d. J., und zwar zuerst an leichter Angina; am 4. April heftige Schmerzen, Sausen und Schwerhörigkeit im rechten Ohre. Trommelfell stark aufgelockert, livid, serös durchfeuchtet; Hörweite sehr vermindert, Flüstersprache wird nicht gehört, Jaute Ansprache nicht über 2 Meter. Knochenleitung vom Scheitel nach rechts verstärkt; Rinne positiv. Körpertemperatur bis zu $38,6^{\circ}$ Abends. Das Trommelfell wurde sogleich am 5. April Morgens ausgiebig incidirt und blutig seröses Secret entleert. Die Behandlung bestand in den folgenden 4 Tagen in Durchspülungen der Paukenhöhle mit Sublimatspiritus (1:1000) mit Politzer's Verfabren und warmen Cataplasmen. Dabei verschwanden die Schmerzen sehr bald, die schleimigseröse Secretion hörte anf, die Hörweite hob sich rasch und betrug bereits am siebenten Tage nach der Paracentese 2 Meter für Flüstersprache, am 8. Tage war die perforationsöffnung geschlossen. Geringe Fieberbewegung war noch $\mathrm{zn}$ constatiren. Aber das Allgemeinbefinden entsprach nicht der fortschreitenden Heilung der Otitis. Hartnäckige Stuhlverstopfung, Anorexie, erneute Fiebersteigerung bis $\mathrm{zu} 39,5^{\circ}$ Abends, heftige Schmerzen in den verschiedensten Körperthellen, besonders im Nacken und Rücken, quälten den Kranken, obne dass man noch eine anderweitige Organerkrankung nachweisen konnte, man musste eine schwere Complication befürchten. Am neunten Tage nach Beginn der Ohrerkrankung endlich zeigte sich deutlich Schwellung des rechten Kniegelenkes. Alsdann warden der Reibe noch die meisten ubrigen Gelenke befallen und schliesslich auch die Halswirbelverbindungen. Während die entzündliche Affection jedes einzelnen Gelenkes nach kurzer Dauer wieder schwand, entwickelte sich ein umfangreiches Exsudat im Pericardium und dann in der rechten Pleurahöhle. Mehrmals trat Herzschwäche ein. welche Campherinjectionen nöthig machte (Puls irregulär, bis za 124 in der Minute). Dabei magerte der Kranke ausserordentlich $a b$ und blieb sehr hinfällig bis zur 9. Krankheitswoche, nach welcher allmählich Erboblung eintrat. Ein Jängerer Aufenthalt auf dem Lande fuhrte dann zur vollea Reconvalescenz. Seitens des Ohres waren vom Patienten Störungen nicht mehr bemerkt worden, doch ich konnte bei der letzten Unter- 
suchung am 23. October d. J, folgendes feststellen: Das rechte Trommelfell erscheint entschieden sehnig verdickt, besonders in der hinteren Hälfte, Lichtkegel verstrichen, Hammergriff und Proc. brev. mehr hervortretend als links und dicker in den Contonren, Hörweite etwa 6 Meter für Flïsterworte, welche links noch uber 12 Meter hinaus nachgesprochen werden.

Die beiden Fälle zeigen übereinstimmend die nach Ablauf der rheumatischen Affection zurickgebliebene Verdichtung der Paukenhöhlenauskleidung, welche sich aus der acuten Otitis entwickelt hatte. Wir haben somit, wenigstens für einen Theil der Fälle von sogenannter Sklerose, deren erste Entstehung verfolgen können, und die Bezeichnung ", r h e m at is che O tit is " möchte vielleicht ebenso berechtigt sein, wie die seiner Zeit von mir vorgeschlagene Benennung Otitis media diabetica seu necrotica für die beim Diabetes sich entwickelnde acute Form.1) Die rheumatische Otitis acuta unterscheidet sich besonders durch ihren verbältnissmässig milden und raschen Verlauf sehr wesentlich von der acuten eitrigen Otitis, wie wir sie u. A. in Influenza- und Scharlachepidemien und bei Diabetes auftreten sehen. Für die Beurtheilung einer bei Otitis media acuta auftretenden Complication erscheint es jedenfalls geboten, sich gegenwärtig zu halten, dass erneute Temperatursteigerung, Kopf- und Nackenschmerzen nicht immer auf eine Gehirncomplication hindeuten, sondern auch den Beginn eines schweren, acuten Gelenkrheumatismus anzeigen können, ebenso wie zuweilen eine Otitis media dem Ausbruch des Scharlach-Exanthems vorausgeht. Die nevere Literatur uiber den acuten Gelenkrheumatismus ${ }^{2}$ ) betont das häufige Vorkommen der prodromalen Angina. Man könnte daher meinen Erörterungen gegenüber einwenden, dass die von mir beschriebene Form der Otitis nicht als Fribsymptom oder Theilerscheinung der Polyarthritis acuta aufufassen sei, sondern einfach als eine von der prodromalen Angina per tubam fortgeleitete Entzündung der

1) Vgl. Tageblatt der 59. Vorsammlung deutscher Naturforscher and Aerzte zu Wiesbaden 1887.

2) Buss, Ueber die Beziehungen zwischen Angina und acutem Gelenkrheumatismus. Deutsches Archiv für klinische Medicin. Bd. 54. 1. 1894. Roos, Ueber rhenmatische Angina (Polyarthritis anginosa). Berliner klin. Wochenschrift. Nr. 25, 26. - Su channek, Die Beziehungen zwischen Angina und acutem Gelenkrheumatismus. Sammlung zwangloser Abhandlungen aus dem Gebiete der Nasen-, Ohren-, Mund- und Halskrankheiten. Heft 1. 1895. - Gerhardt, Vortrag am 8. April 1896 auf dem Congress f. innere Medicin zu Wiesbaden über "Rheumatoid". - Fiedler, Zur Aetiologie der Pleuritis. Jahresbericht der Gesellschaft für Natur- und Heilkunde in Dresden. $1890-1891$. 
Paukenhöhle. Im ersten meiner Fälle war aber eine Angina nicht vorausgegangen, im zweiten war dieselbe sehr leicht und bei Einsetzen der Otitis kaum mehr ausgesprochen; uberhaupt ist der Procentsatz der prodromalen Angina nach den oben citirten Autoren kein sehr hoher und noch nicht definitiv festgestellter. Die essentielle Angina tonsillaris giebt uberhaupt seltener Veranlassung zu wirklicher Otitis media acuta, als zu einfachem Tubenverschluss.

Grödel 1) gesteht der prodromalen Angina bei Polyarthritis nur die Bedeutung einer Localaffection zu, gewissermaassen bilde sie die Eingangspforte, von welcher aus die zur Erregung einer Rheumarthritis geeigneten Kokken den Weg in das Innere des Körpers nehmen. In unseren Fällen haben die specifischen Infectionsträger des Rheumatismus wohl eher auf dem Wege der Blutbahn, vielleicht auch direct per tubam die erste Localisation in der Paukenhöhle und in den Gelenken der Gehörknöchelchen bewirkt.

Für den Bacteriologen wäre es vielleicht lohnend, beim Forschen nach den Mikroben der Polyarthritis direct nach der Paracentese des Trommelfelles das Secret der Paukenhöhle zu entnehmen, dasselbe ist in dem ersten Stadium dieser Otitis rein und leicht qu haben.

Nachtrag.

Nachdem Adolf M. bei der letzten Untersuchung am 23. Oct. d. J. sich mir als blithend und gesund vorgestellt hatte, befiel ihn am 6. November ein Recidiv des Gelenkrheumatismus ohne prodromale Angina und ohne Störungen seitens des Ohres. Bei einer Abendtemperatur bis zu $39,4^{\circ} \mathrm{C}$. war das rechte Schultergelenk schmerzhaft und geschwellt, dazu trat wiederum ein Exsudat in die rechte Pleurahöhle. Die Erkrankung verlief jedoch sehr gïnstig, und schon nach 10 Tagen war Genesung eingetreten. Diese nachträgliche Mittheilung erschien mir deshalb von differentielldiagnostischem Werthe, weil sie den etwaigen Einwurf, als ob die weiter oben geschilderte multiple Gelenkaffection vielleicht doch als eine pyämische ex otitide hätte aufgefasst werden können, vollständig beseitigt, auch abgesehen von allen anderen Gründen, welche gegen die pyämische Form und für echten, genuinen Gelenkrheumatismus sprachen.

1) Ueber acuten Gelenkrheumatismus in Anschluss an Angina von Dr. Grödel in Bad Nauheim. Deutsche medicinische Wochenschrift. 1896. Nr. 17. 